\title{
Photonic Computing with Single and Coupled Spiking Micropillar Lasers
}

\author{
V. A. Pammi, K. Alfaro-Bittner, M. G. Clerc, S. Barbay \\ Invited Paper
}

\begin{abstract}
We review experimental and theoretical results on the computing properties of single spiking micropillar lasers and present numerical studies of propagation and computing in chains of evanescently coupled micropillar lasers. Single micropillar lasers are shown to behave as ultrafast optical neurons with sub-nanosecond spike times. They also possess absolute and relative refractory times, spike latency and show temporal summation. With delayed optical feedback, they emulate an autapse. These basic neural properties can be used for simple photonic computing. We show by numerical simulations of a chain of coupled spiking micropillar lasers that basic logical operations can be implemented in photonic circuits, as well as temporal pattern recognition based on the collision properties of pulses in this chain.
\end{abstract}

Index Terms-neuromimetic photonics, laser with saturable absorber, excitability, spiking

\section{INTRODUCTION}

$\mathbf{N}$ EUROMIMETIC photonic systems have attracted a lot of attention lately because of their potential for applications in analog, Non-Von Neumann and spike based computing schemes [1], [2]. One of the most prominent property of biological neurons is their ability to respond in an all-or-none fashion to input perturbations in the form of a well-defined electrical spike. This phenomenological response is called excitability. In the brain, information is encoded into spikes which are also used for computing. Different semiconductorbased photonic systems have been proposed to mimic the response of biological neurons with spikes of light [3], [4], [5], [6], [7], [8], [9], [10], [11]. Many of these systems rely on coherent injection of a laser cavity. In 2011 we proposed the first excitable semiconductor laser with an integrated saturable absorber (SA). This system has the advantage of needing only incoherent pumping (either optical or electrical) and presents a slow-fast non-linearity based on carriers rather than on a slow thermal response. The micropillar lasers [12] can be integrated easily into arrays for further coupling either through free-space or spatial schemes (evanescent coupling [13], [14]). It is thus promising to design complex architectures using

V. A. Pammi is with the Centre de Nanosciences et de Nanotechnologies, CNRS-UPSud-UPSaclay, Palaiseau, France, e-mail: anirudh.pammi@c2n.upsaclay.fr.

K. Alfaro-Bittner is with the Instituto de Física, Pontificia Universidad Católica de Valparaíso, Valparaíso, Chile , e-mail: karin.alfaro.b@mail.pucv.cl.

M. G. Clerc is with the Departamento de Física and Millenium Institute for Research in Optics, Facultad de Ciencias Físicas y Matemáticas, Universidad de Chile, Santiago, Chile, e-mail: marcel@dfi.uchile.cl.

S. Barbay is with the Centre de Nanosciences et de Nanotechnologies, CNRS-UPSud-UPSaclay, Palaiseau, France, e-mail: sylvain.barbay@c2n.upsaclay.fr. several nonlinear units and could be used as a building block for photonic spiking artificial neural networks.

In the following, we first review our recent results on the neuromimetic and computing properties of single and delaycoupled micropillar laser with an integrated saturable absorber. We then present original results on the computing ability of evanescently-coupled excitable micropillar nodes and evidence spike-based logical gates on-chip circuits as well as temporal spike pattern recognition. These circuits rely on the collision properties of spikes and on integrated delays in spatially coupled micropillar lasers.

\section{EXCITABLE MICROLASER}

The excitable system we consider is a micropillar laser with an integrated saturable absorber. It follows an original structure based on a vertical-cavity microlaser with integrated saturable absorber, with optimized AlGaAs/AlAs aperiodic multilayer mirrors allowing for efficient optical pumping over a large wavelength window [15], [16]. The active zone of the microcavity contains two InGaAs/GaAs gain quantum wells and one InGaAs/AlGaAs SA quantum well, each placed at the anti-node of the cavity resonance field targeted at $980 \mathrm{~nm}$. The SA quantum well is located at a node of the pump field, and its wavelength is $\simeq 800 \mathrm{~nm}$. This vertical-cavity microlaser with integrated saturable absorber has been shown to display a fast excitable response [10], [12], with 200 ps spike times. This is to be compared to the millisecond timescale of biological neurons response or the timescale found in electronic systems ranging from microsecond to nanosecond at best in FGPAbased spiking approaches [17]. The structure is then etched to design a single node with a $4 \mu \mathrm{m}$ diameter and coated with a $\mathrm{SiN}$ layer to protect from oxidation and improve the thermal dissipation. The final micropillar is depicted in Fig.1.

We characterize the excitable behavior by studying the response of the system to coherent perturbations sent close to the cavity resonance [18]. Fig.2 shows the response of the micropillar laser in the excitable regime subjected to a train of perturbation pulses with duration $80 \mathrm{ps}$ and repetition rate $82 \mathrm{MHz}$. The perturbation pulses are produced using a modelocked Ti:Sa laser operating close to the micropillar resonance $(\sim 980 \mathrm{~nm})$. The micropillar laser is pumped in the excitable regime, just below its self-pulsing threshold [19]. We clearly identify a threshold below which the perturbation pulses do not produce a macroscopic response, whereas above this threshold they produce a calibrated response. The response amplitude in that case does not depend on the perturbation amplitude, and has a width of $\sim 200$ ps. This is a clear manifestation of the 


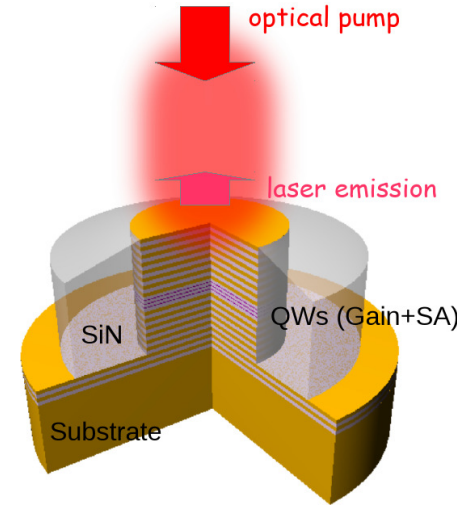

Fig. 1. Sketch of the micropillar laser with intracavity SA structure. The design is optimized for an optical pump wavelength around $800 \mathrm{~nm}$ and the emission wavelength is targeted at $980 \mathrm{~nm}$. See text for the description of the structure. The micropillar diameter is $4 \mu \mathrm{m}$.

phenomenon of ultrafast excitability. Close to the excitable threshold, some pulses are excited because of the noise. Away from the excitable threshold, the response of the system is deterministic and a macroscopic excitable pulse is generated for each perturbation pulse.
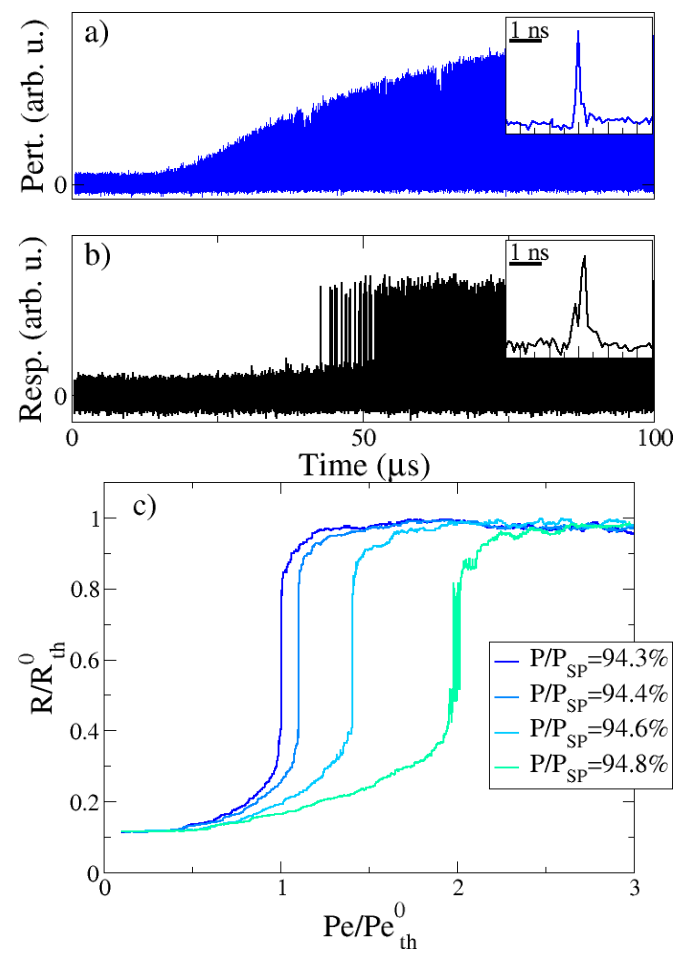

Fig. 2. Response of the micropillar laser in the excitable regime. a) Train of 80 ps perturbation pulses at $82 \mathrm{MHz}$ repetition rate with increasing amplitude. Inset: zoom on a single perturbation pulse. b) Response pulses of the micropillar in the excitable regime. Inset: zoom on a single response pulse. c) Median average of the scaled response $R$ versus the scaled perturbation pulse amplitude $P e$ for different bias pumps $P$ w.r.t the self-pulsing laser threshold $P_{S P}$.

This is confirmed by the plot of the response versus perturbation amplitude in Fig.2c, for different bias pumping values represented by different colors. There is an abrupt transition in the response below and above the excitable threshold, and a strong clamping of the response amplitude above threshold. This behavior has been confirmed by a theoretical analysis of the system of equations governing the dynamics [20]. It is also interesting to note that the excitable threshold can be controlled with the bias pumping value. This was also the case for incoherent perturbations sent in the pump window, close to $800 \mathrm{~nm}$ [12]. However, in the coherent perturbation case, there are intricate effects of thermal-induced change of cavity resonance wavelength that hinders the theoretical expectations of an increase of the excitable threshold for lower bias pump values. The threshold for exciting a response was measured to be on the order of few fJ for coherent and $\sim 700 \mathrm{fJ}$ for incoherent perturbations. The response energy was estimated to be of the order of $50 \mathrm{fJ}$ [18]. Note that incoherent perturbations are much less energy efficient than coherent ones. This is essentially due to the fact that incoherent perturbations are almost transverse to the unstable direction of the stable, laseroff state. However, cascadability is ensured through coherent re-injection and is thus possible and energetically efficient.

Another major property associated with excitable behavior is the presence of refractory periods. These refer to the response of the system subjected to consecutive, above threshold, perturbations. It is well known in (integrator-type) excitable systems that there exists a period of time immediately following the firing of an excitable pulse during which it is not possible to excite another pulse. This is called the absolute refractory period, and is linked to the excitable spike duration. It was measured to be around 200 ps [12]. However, after this period of time the system has not usually recovered completely. There is thus a second period of time during which it is usually more difficult to elicit a spike because the excitable threshold is increased. This is called the relative refractory period. It has also been measured in the micropillar system around 350 ps [12]. Both timescales are intimately linked to the internal timescales of the system, and in particular to the recovery of the carriers in the semiconductor material. In the micropillar laser, the photon dynamics is much faster than the carrier dynamics. Therefore, after a spike has been fired, the system takes time to recover to its steady state. The recovery time and the relative refractory period have been explained in terms of the net gain dynamics, i.e. the recovery dynamics of the gain and loss in the cavity. This mechanism introduces a memory in the system, which remembers through the carrier variable of its previous state. These timescales are extremely important in many computational applications, in particular for the coincidence detector neuron and for the propagation of excitable pulses in coupled microcavity chains.

In micropillar lasers, excitability follows a homoclinic-loop bifurcation [21], [19]. There is thus a critical point and a threshold associated with this behavior. It is well known in that case that we expect a delay in the response, of nonlinear origin. This delay has been measured to be of the order of a few 100s of ps and it depends on the above-threshold amplitude of the perturbation pulse [18]. It has been shown to introduce very naturally a temporal-coding mechanism for the input stimuli. Indeed, while it was usually believed that coding of information in the brain was made through the rate of spikes, it has been shown in 2001 [22], [23] that this coding 
scheme could not explain some behavioral data on image processing by the brain and that probably information had to be coded in only one spike. In the temporal coding scheme, an input stimulus of strong amplitude and above the excitable threshold will produce a fast response, whereas a stimulus just above threshold will produce a spike following a long latency time, therefore realizing a temporal coding of input stimuli. The spike latency time can be computed and shows a very nonlinear dependence with the input perturbation [19], [18], [20]. Spike latency time has been found also in biological neurons [24], [25] and is thus part of the computation process which takes place in the brain.

\section{COMPUTING WITH SINGLE NODE}

Whereas the computation power of the brain stems from the numerous neurons and connections present in it, it is fascinating to realize that a single neuron can process information [26], [27]. As was noticed in [28], our micropillar laser with integrated saturable absorber can act as a leaky integrate-and-fire (LIF) neuron, a neuron model widely used in computational neuroscience. A LIF neuron has several inputs (to mimic the dendritic tree), and a soma which sums, integrates and compares the integrated inputs to a threshold. In the LIF neuron model, only the subthreshold current is modeled. When the excitatory postsynaptic potential reaches the excitable threshold, a spike is (formally) emitted and the neuron excitation is reverted to zero. In principle, this model does not account for refractory periods but it can be accounted for in variants of the model.

The micropillar laser with integrated SA does show the summation and integration properties. To test this, the micropillar was subjected to delayed, sub-threshold stimuli. Each stimulus by itself is not sufficient to elicit a spike. When the two stimuli are sent far away apart, the situation is similar to sending only one stimulus. However, when the stimuli are sent within a small time window, they can sum up and cross the excitable threshold, producing an excitable pulse. This phenomenon was demonstrated with two delayed input pulses in the micropillar laser [29]. Here again, the relative refractory period is the relevant timescale for the summation to take place. This also demonstrates that the micropillar laser can detect the coincidence of sub-threshold incoming pulses when their delay in below the refractory period, i.e. below $\sim 350 \mathrm{ps}$. This confirms that the micropillar laser with integrated SA has the properties of a LIF neuron, but is a more complex system since it possess also refractory periods and emits nonlinear spikes.

Another major phenomenon related to computing is memory storage. It has been shown that excitable systems with delayed feedback can give rise to regenerative pulsing [30], [31]. This provides the basis of a regenerative memory for temporal spike patterns. A micropillar laser with delayed optical feedback was shown experimentally to behave analogously [32], allowing the fast optical control with single input perturbations of the optical buffer of spikes. Both repulsive and attractive pulse to pulse interaction were evidenced. A numerical and theoretical analysis concluded that pulse interaction (attractive and/or repulsive) should lead to asymptotic states consisting of regular spiking patterns. In the presence of noise (pump noise in particular), it was shown that the induced stochasticity in the dynamics is biased towards the erasure of spikes [33]: noise plays an asymmetric role. It is more likely to prevent the regeneration of a pulse than creating a pulse from nothing. There are certain times where the system is more sensitive to noise. From the dynamical point of view, this system illustrates a non standard escape process from a fixed point to a limit cycle. From a spike-based computing perspective, this behavior indicates how the system can be robust versus noise, which is an inherent part of the nervous system [34], by not creating artefacts. It also indicates in this specific case how noise, by limiting the memory time, induces a natural fading memory which is an essential ingredient of e.g. reservoir computing with recurrent neural networks [35].

\section{SPATIALLY COUPLED MiCROPILlaR CHAiN}

For compacity reasons, it is interesting to explore how one could leverage photonic spiking systems to build integrated circuits and networks. Important elements of such networks are the ability to link the network nodes and to cascade the different functionalities, the introduction of delays and of weights. Coupled excitable nodes have been theoretically and experimentally studied in the past. Experimental studies include semiconductor quantum-dot lasers using free-space coupling [36] and excitable microring lasers coupled through waveguides [11]. Theoretical studies have been carried out in coupled waveguides and optically injected microdisk lasers [37]. We propose in the following a different approach and study spatially coupled neuromimetic micropillar lasers in order to analyze some computing properties of such networks.

The dynamics of a chain of $n$ evanescently coupled micropillar lasers can be modeled by a set of $3 \times n$ coupled ODEs [38], [39]:

$$
\begin{aligned}
& \dot{E}_{i}=\left((1-i \alpha) G_{i}-(1-i \beta) Q_{i}-1\right) E_{i}+i \kappa\left(E_{i-1}+E_{i+1}\right) \\
& \dot{G}_{i}=b_{1}\left(\Lambda-G_{i}\left(1+\left|E_{i}\right|^{2}\right)\right) \\
& \dot{Q}_{i}=b_{2}\left(\gamma-Q_{i}\left(1+s\left|E_{i}\right|^{2}\right)\right)
\end{aligned}
$$

The dynamical variables for the i-th microcavity are the envelope of the electric fields $E_{i}$, the rescaled gain $G_{i}$ and the rescaled absorption $Q_{i}$. Time is rescaled to the cavity photon lifetime which is experimentally of the order of several ps (12 ps). A dispersive nearest-neighbour coupling coefficient $\kappa$ between the micropillars is introduced. It describes the effect of the mode overlap between the cavities and is supposed to be real in order to conserve the energy. Non-radiative carrier recombination rates for the gain and absorber are $b_{1}$ and $b_{2}$, and the alpha enhancement factors are $\alpha$ and $\beta$, respectively. There is a pump term $\Lambda$ for the gain, whereas non-saturable losses are represented by $\gamma$. The saturation parameter is $s=a_{2} b_{1} / a_{1} b_{2}$, with $a_{1,2}$ the differential gain and absorption, controls the characteristic response of the system. Excitability requires that $s>1+1 / \gamma$, which is usually the case in semiconductor materials since $s$ is a large parameter because of the gain saturation. The laser threshold for the 
single microlaser with $\mathrm{SA}$ is $\Lambda_{\mathrm{th}}=1+\gamma$. In the excitable regime, for $\Lambda<\Lambda_{t h}+\kappa$, an initial perturbation above the excitable threshold can propagate to the neighboring cavities in the saltatory propagation regimeprovided $\kappa \ll 1$. Indeed, in this regime the coupling time is large as compared to the photon cavity lifetime which ensures that an excitable response can form before the energy couples to the neighboring cavities. Note also that the model implicitly assumes that the coupledmode theory is valid, i.e. that the coupling strength is small enough too. We will also consider $b_{1}, b_{2} \ll 1$ since the carrier non-radiative recombination times are much longer than the photon lifetime in the semiconductor structure considered.

Figure 3 shows the saltatory propagation of an excitable response in the micropillar chain. The parameters are compatible with semiconductor parameters [40] and are taken as $b_{1}=b_{2}=0.001, \gamma=2, s=10, \Lambda=2.8, \kappa=0.1$, $\alpha=2, \beta=0$. This corresponds to physical non-radiative recombination timescales of 1-2ns which are standard for semiconductor materials. Numerical simulations of the mode structure of coupled micropillars [41] allow to estimate that the coupling strength can be achieved with 4 micron diameter micropillars separated by 3.75 microns. The initial conditions are taken as $G_{i}=\Lambda, Q_{i}=\gamma$ for all cavities, $E_{i \neq 0}=0$ and $E_{0}=F_{0}$. Note that the phenomenon is robust and is observed in a large range of parameters. The leftmost cavity is coherently perturbed by a pulse amplitude $F_{0}=5$, sufficient to elicit an excitable response of the first cavity. Because of the micropillar coupling, the excitable response pulse is coupled to the neighboring cavity and since the coupling strength is sufficient, the excitable threshold is crossed again leading to another pulse. The excitation transfer continues giving rise to a solitonic and ballistic response. Importantly, the response is unidirectional: the excitation can only transfer to the nearest neighbor on the right, or more precisely to the unperturbed cavity. Indeed, a microcavity having fired an excitable pulse will be in the refractory period and will not be able to fire again. Thus, there is a symmetry breaking between the right and left neighbor leading to unidirectional spike propagation.

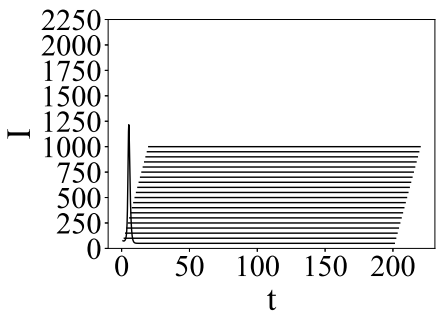

(a)

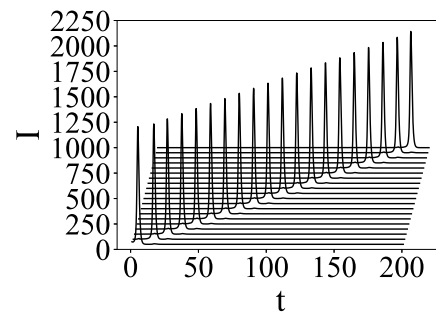

(b)
Fig. 3. Perturbation above excitable threshold of the leftmost cavity (\#1) in a chain of 20 coupled cavities. In (a) and (b), the temporal response of each cavity is plotted with an offset for clarity. The coupling strength in (a) is $\kappa=0.01$ and is too small to induce propagation: only the first cavity fires an excitable spike. In (b), the coupling strength $\kappa=0.1$ is sufficient to induce stable saltatory propagation in the chain.

The calculated mean speed of the pulse for $\Lambda=2.74$ is plotted on Fig. 4a. The speed varies linearly with the coupling strength in a large window of parameters. The speed is very fast and would correspond to propagating across 20 cavities in $200-400$ ps.Below $\kappa \simeq 0.1$, no pulse can propagate. Only when the coupling strength approaches large values that the mean speed saturates and deviates from linearity (not shown), but in this case the validity of the model can be questionable. This relation can be understood physically as follows. The coupling strength controls the time it takes for the energy to be coupled to nearby cavities. When the excitable pulse amplitude is large, and for sufficient coupling strength, the excitable threshold is easily reached. Hence the latency time does not play a large role and the propagation speed does not depend on it. The propagation speed then only depends on the inverse of the coupling time, i.e. on $\kappa$. Note also that the speed is calculated by considering that the distance between the micropillars does not depend on the coupling strength. In practice, this is not exactly true since the coupling strength depends on the overlap integral of the transverse fields inside the microresonators.
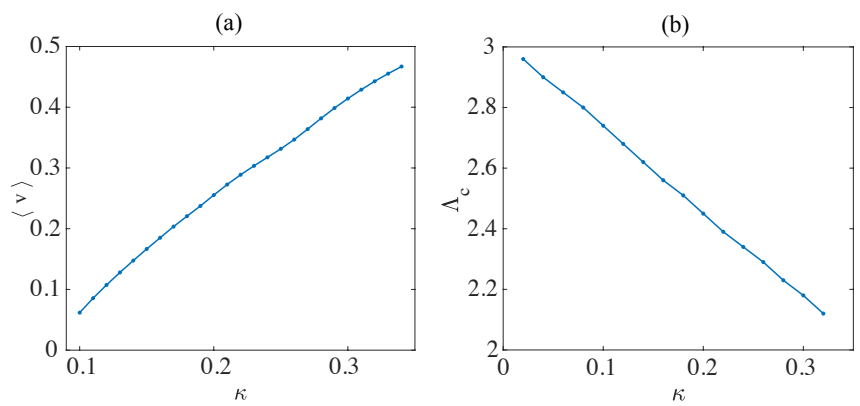

Fig. 4. (a) Mean speed of the pulse $\langle v\rangle$ as a function of coupling strength $\kappa$. (b) Critical pump intensity $\Lambda_{c}$ as a function of coupling strength $\kappa$.

For a given coupling strength $\kappa$ there is a minimal pump intensity value $\Lambda_{c}$ for which propagation is possible. This is illustrated on Fig. 4b. This critical pump value decreases with the pump intensity. Indeed, small coupling strengths must be compensated by higher excitable pulse amplitude, and this is only possible by increasing the pump. This is consistent with the numerical and analytic findings of Refs [18], [20] where the maximum excitable pulse intensity scales as $b_{1}^{-1} \times(\Lambda-$ $1-\ln (\Lambda))$.

\section{SPIKE PROCESSING CIRCUITS}

Spike processing circuits can be readily realized using the previously studied coupled micropillar lasers. In order to illustrate some basic computing abilities, we focus first on the implementation of logic gates for spike-coded information and based on the relative spike timing. Then we demonstrate the effectiveness of coincidence detector neurons for efficient on-chip processing by studying a demonstrator doing temporal spike pattern recognition. We note that the ingredients at stake demonstrated here could serve in a brain-inspired circuit. For instance, a ring recurrent neural network [42] can be build straightforwardly from coupled micropillars and could be used as an echo state network.

\section{A. Excitable logic circuits}

Boolean logic operations with excitable systems have already been studied in models of dendritic spines [43], using 
chemical excitable waves and their collision properties in 2D media [44], [45] and in semiconductor media with excitable localized states [46], [47]. Here we present two logic circuits based on our previous results. In these circuits, information is coded in the presence or absence of a spike and can be represented by two bits, 1 and 0 . These circuits are also designed to be cascadable. A simple OR gate can be constructed with the previous building blocks. We consider a chain with an odd number of microcavities $(n=13)$ for the main segment, to which is attached an additional segment in the center consisting of a single cavity.

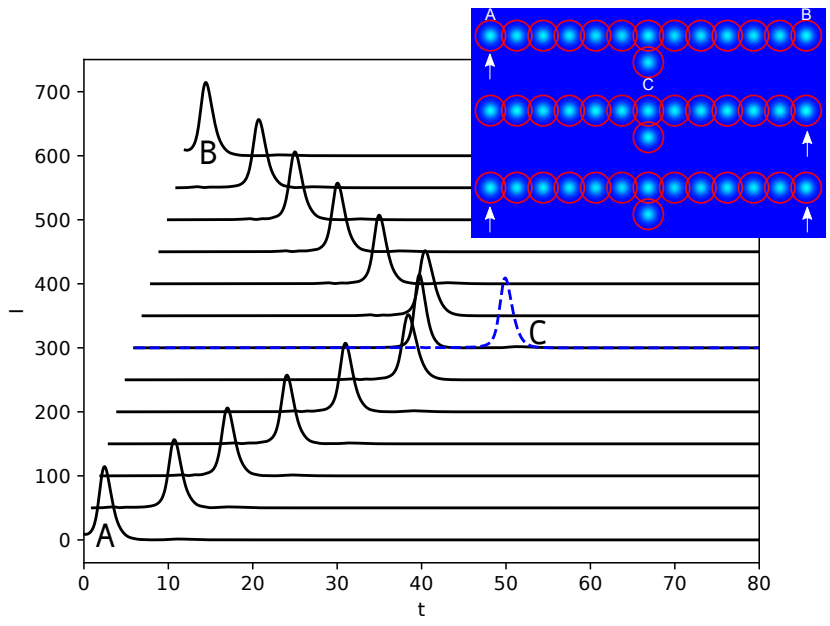

Fig. 5. Numerical simulation of a spike-based OR gate circuit: temporal responses for each cavity (offset for clarity) in the case of two perturbations in $\mathrm{A}$ and $\mathrm{B}$. The response in $\mathrm{C}$ is plotted in dashed and blue. Inset: overlayed responses for inputs in A, B and both A and B. The cavities are pumped with $\Lambda=2.74$. The coupling strength is $\kappa=0.15$.

The OR gate is illustrated on Fig. 5. The two input ports are in A and B. The output port is in C. The above threshold perturbations are marked by arrows. Except in the case where none input is present, the output of the gate in $\mathrm{C}$ is 1 . With only one input, the response is trivially 1 and all the pulses occur after the same amount of time. If the two inputs are 1 , the pulses collide in the central cavity but can excite the output cavity $\mathrm{C}$. Therefore, a pulse emerges and can propagate to possibly another gate. This forms an OR gate. Note that where the two incoming pulses meet, the response latency time is smaller.

The AND gate is less trivial and requires to modify either the coupling or the pumping of the cavities. Since the latter is easier to implement experimentally, we choose this option for the numerics. As was noticed earlier, there is a critical pump for the spike to propagate. We use this property to build the AND spike-based gate. We consider the same chain as before, except the microcavities immediately next to the central cavity are now pumped with a lower value (see Fig. 6). The value is chosen to prevent the propagation of a single pulse. However, when the two inputs are present, the coupling of the two lower intensity pulses in the central cavity is sufficient to cross the excitable threshold and a pulse is created in C. The lower pump is immediately translated into a larger spike latency time and a smaller response pulse. When these smaller pulses are coupled

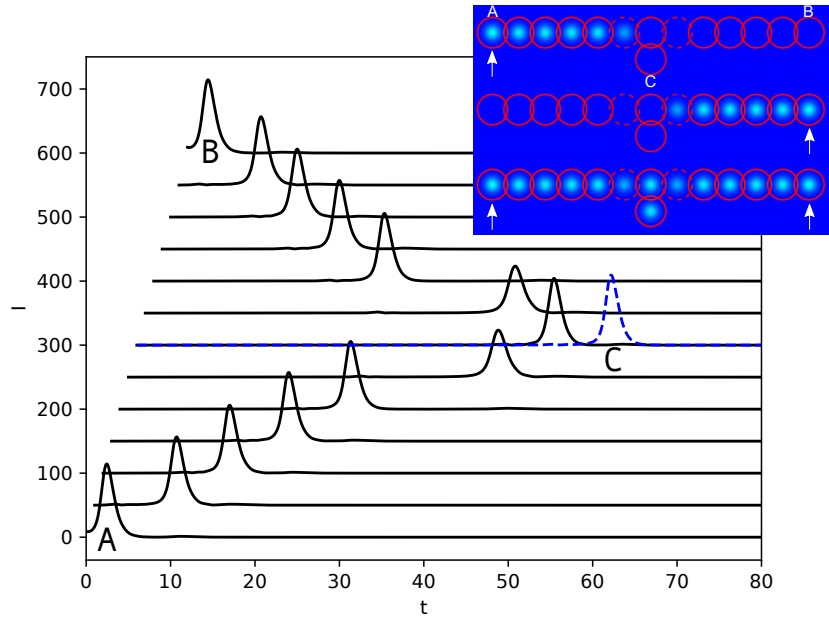

Fig. 6. Numerical simulation of a spike-based AND gate circuit: temporal responses for each cavity (offset for clarity) in the case of two perturbations in $\mathrm{A}$ and $\mathrm{B}$. The response in $\mathrm{C}$ is plotted in dashed and blue. Inset: overlayed responses for inputs in A, B and both A and B. The cavities are pumped with $\Lambda=2.74$ except the one marked by dashed circles with $\Lambda^{\prime}=2.45$. The coupling strength is $\kappa=0.15$.

into the central cavity, they can produce a response pulse in $\mathrm{C}$ which can be cascaded eventually to other gates. The same phenomenon arises for a constant pump value of $\Lambda=2.74$ and a change of the coupling constant of the nodes next to the vertex node, for values in the range $0.05 \lesssim \kappa^{\prime} \lesssim 0.1$. If $\kappa^{\prime} \lesssim 0.05$, the pulse cannot propagate anymore and is stopped completely. If $\kappa^{\prime} \gtrsim 0.1$, the gate transforms into an OR gate since a single pulse can propagate through the vertex.

\section{B. Temporal spike pattern recognition}

A simple circuit illustrating the temporal pattern recognition of two spikes separated by $\Delta t=515.5$ is shown on Fig. 7. The input signal is sent in $A$ and is recorded in several places of the circuit. The output node is in $D$. The input spike propagates through the upper arm (see inset in Fig. 7). At the first crossing, the signal is split in two parts. The lower arm implements a longer delay. Both arms meet in a node structure similar to the previously shown AND gate. Every time the temporal pattern in input is recognized, a spike is emitted in $D$. To speed-up the computation time and reduce the size of the delay line we have accelerated the carrier dynamics in the simulation. The input pattern recognized corresponds essentially to the propagation delay of the pulse in the lower arm. Generalization of the circuit to the recognition of more complex temporal patterns is straightforward. However, the refractory time of the nodes sets the lower limit of the detectable separation between two input spikes.

While the topology of the spike pattern decoder is fixed by fabrication, this does not mean that the system has no flexibility at all. Indeed, it would be possible to modulate the pumping intensity in one of the arms to change the on-chip delays and to recognize different temporal patterns. In the experiment this would be possible for instance by incorporating a spatial light modulator on the pump arm to 


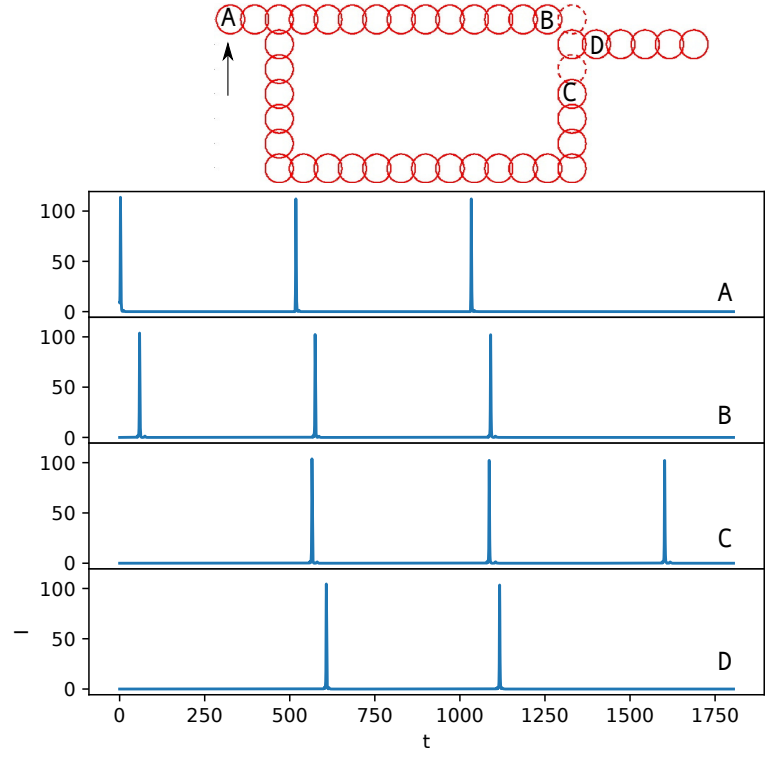

(a)

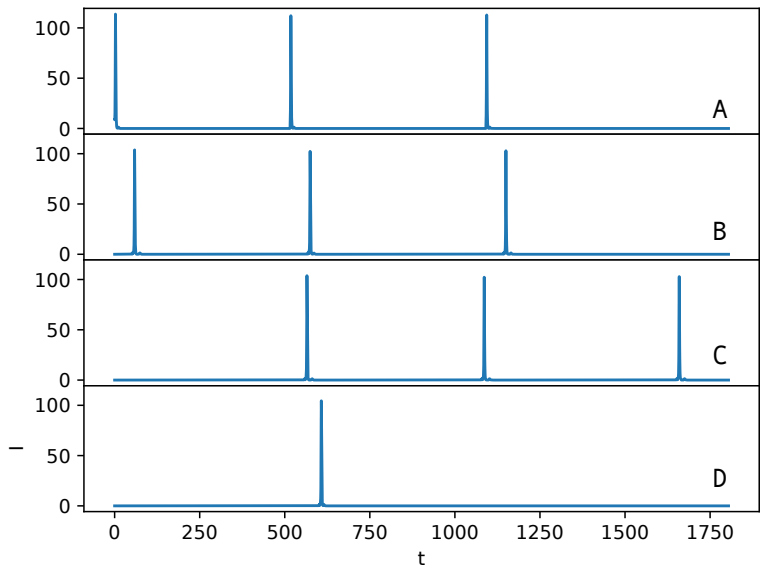

(b)

Fig. 7. Spike-based temporal pattern recognition. A signal is sent in $A$ and recorded in $A, B, C$ and $D$. In $D$, the output pulse signals the presence of specific temporal pattern: two spikes separated by $\Delta t=515.5$. In (a) the input signal consists of three spikes separated by $\Delta t$. In (b) the input signal consists of two first spikes separated by $\Delta t$ and a third spike separated by $\Delta t^{\prime}=575.5$. In inset is shown the micropillar circuit considered. The micropillar are uniformly pumped except the two pillars with dashed circles (same parameters as in Fig. 6 except $b_{1}=b_{2}=0.01$ ). Inset: schematic of the circuit used (the lower arm length has been reduced for clarity). The total number of nodes is 105 .

modulate spatially the pump. It would therefore introduce some kind of learning capability to the system by monitoring the output node and changing the pump intensity in the desired arm.

\section{CONClusions}

In conclusion, we have reviewed the computing ability of single and coupled spiking micropillar lasers. We have shown that circuits implementing tunable delays and pulse collisions can be constructed to perform temporal recognition tasks. These systems can also serve as novel substrates for ultrafast artificial neural networks, implementing on-chip delays and weighted connections, by combining the different properties evidenced here in this system.

\section{ACKNOWLEDGMENT}

S.B. and V.A.P. acknowledge support from the CNRS Renatech network of cleanroom facilities as well as from the European Union's Horizon 2020 Research and Innovation Program (Fun-COMP project, \#780848). M.C. acknowledges financial support from the Millennium Institute for Research in Optics (MIRO) and Fondecyt under Grant No. 1180903. K.A.B. is supported by CONICYT, scholarship Beca Doctorado 2014 Nacional No. 21140668.

\section{REFERENCES}

[1] P. R. Prucnal, B. J. Shastri, T. F. de Lima, M. A. Nahmias, and A. N. Tait, "Recent progress in semiconductor excitable lasers for photonic spike processing," Advances in Optics and Photonics, vol. 8, no. 2, p. 228, may 2016.

[2] T. F. de Lima, B. J. Shastri, A. N. Tait, M. A. Nahmias, and P. R. Prucnal, "Progress in neuromorphic photonics," Nanophotonics, vol. 6, no. 3, jan 2017.

[3] M. Giudici, C. Green, G. Giacomelli, U. Nespolo, and J. R. Tredicce, "Andronov bifurcation and excitability in semiconductor lasers with optical feedback," Phys. Rev. E, vol. 55, no. 6, pp. 6414-6418, Jun 1997.

[4] H. J. Wünsche, O. Brox, M. Radziunas, and F. Henneberger, "Excitability of a semiconductor laser by a two-mode homoclinic bifurcation," Phys. Rev. Lett., vol. 88, no. 2, p. 023901, Dec 2001.

[5] S. Barland, O. Piro, M. Giudici, J. R. Tredicce, and S. Balle, "Experimental evidence of van der pol-fitzhugh-nagumo dynamics in semiconductor optical amplifiers," Physical Review E, vol. 68, no. 3, sep 2003.

[6] A. M. Yacomotti, P. Monnier, F. Raineri, B. B. Bakir, C. Seassal, R. Raj, and J. A. Levenson, "Fast thermo-optical excitability in a twodimensional photonic crystal," Physical Review Letters, vol. 97, no. 14, oct 2006.

[7] D. Goulding, S. P. Hegarty, O. Rasskazov, S. Melnik, M. Hartnett, G. Greene, J. G. McInerney, D. Rachinskii, and G. Huyet, "Excitability in a quantum dot semiconductor laser with optical injection," Physical Review Letters, vol. 98, no. 15, apr 2007.

[8] A. Hurtado, I. D. Henning, and M. J. Adams, "Optical neuron using polarisation switching in a 1550nm-VCSEL," Optics Express, vol. 18, no. 24, p. 25170, nov 2010.

[9] S. Beri, L. Mashall, L. Gelens, G. V. der Sande, G. Mezosi, M. Sorel, J. Danckaert, and G. Verschaffelt, "Excitability in optical systems close to z2-symmetry," Phys. Lett. A, vol. 374, no. 5, pp. 739 - 743, 2010.

[10] S. Barbay, R. Kuszelewicz, and A. M. Yacomotti, "Excitability in a semiconductor laser with saturable absorber," Opt. Lett., vol. 36, no. 23, pp. 4476-4478, Dec 2011. [Online]. Available: http://ol.osa.org/abstract.cfm?URI=ol-36-23-4476

[11] T. V. Vaerenbergh, M. Fiers, P. Mechet, T. Spuesens, R. Kumar, G. Morthier, B. Schrauwen, J. Dambre, and P. Bienstman, "Cascadable excitability in microrings," Optics Express, vol. 20, no. 18, p. 20292, aug 2012.

[12] F. Selmi, R. Braive, G. Beaudoin, I. Sagnes, R. Kuszelewicz, and S. Barbay, "Relative refractory period in an excitable semiconductor laser," Phys. Rev. Lett., vol. 112, p. 183902, May 2014, \& Synposis in Physics, Semiconductors Laser Get Nervy. [Online]. Available: http://link.aps.org/doi/10.1103/PhysRevLett.112.183902

[13] A. Dousse, J. Suffczyński, A. Beveratos, O. Krebs, A. Lemaître, I. Sagnes, J. Bloch, P. Voisin, and P. Senellart, "Ultrabright source of entangled photon pairs," Nature, vol. 466, no. 7303, pp. 217-220, jul 2010.

[14] P. St-Jean, V. Goblot, E. Galopin, A. Lemaitre, T. Ozawa, L. L. Gratiet, I. Sagnes, J. Bloch, and A. Amo, "Lasing in topological edge states of a one-dimensional lattice," Nature Photonics, vol. 11, no. 10, pp. 651-656, sep 2017. 
[15] T. Elsass, K. Gauthron, G. Beaudoin, I. Sagnes, R. Kuszelewicz, and S. Barbay, "Fast manipulation of laser localized structures in a monolithic vertical cavity with saturable absorber," Appl. Phys. B, vol. 98, pp. 327-331, 2010, 10.1007/s00340-009-3748-9. [Online]. Available: http://dx.doi.org/10.1007/s00340-009-3748-9

[16] _ "Control of cavity solitons and dynamical states in a monolithic vertical cavity laser with saturable absorber," Eur. Phys. J. D, vol. 59, pp. 91-96, 2010, 10.1140/epjd/e2010-00079-6. [Online]. Available: http://dx.doi.org/10.1140/epjd/e2010-00079-6

[17] D. P. Rosin, D. Rontani, and D. J. Gauthier, "Ultrafast physical generation of random numbers using hybrid boolean networks," Physical Review E, vol. 87, no. 4, apr 2013.

[18] F. Selmi, R. Braive, G. Beaudoin, I. Sagnes, R. Kuszelewicz, T. Erneux, and S. Barbay, "Spike latency and response properties of an excitable micropillar laser," Phys. Rev. E, vol. 94, p. 042219, Oct 2016. [Online]. Available: http://link.aps.org/doi/10.1103/PhysRevE.94.042219

[19] J. L. A. Dubbeldam, B. Krauskopf, and D. Lenstra, "Excitability and coherence resonance in lasers with saturable absorber," Phys. Rev. E, vol. 60, no. 6, pp. 6580-6588, Dec 1999.

[20] T. Erneux and S. Barbay, "Two distinct excitable responses for a laser with a saturable absorber," Phys. Rev. E, vol. 97, no. 6, jun 2018.

[21] J. L. A. Dubbeldam and B. Krauskopf, "Self-pulsations of lasers with saturable absorber : dynamics and bifurcations," Opt. Commun., vol. 159, p. 325,1999

[22] S. Thorpe, A. Delorme, and R. V. Rullen, "Spike-based strategies for rapid processing," Neural Networks, vol. 14, no. 6-7, pp. 715-725, jul 2001.

[23] R. V. Rullen and S. J. Thorpe, "Rate coding versus temporal order coding: What the retinal ganglion cells tell the visual cortex," Neural Computation, vol. 13, no. 6, pp. 1255-1283, jun 2001.

[24] A. I. Wlodarczyk, C. Xu, I. Song, M. Doronin, Y.-W. Wu, M. C. Walker, and A. Semyanov, "Tonic GABAA conductance decreases membrane time constant and increases EPSP-spike precision in hippocampal pyramidal neurons," Frontiers in Neural Circuits, vol. 7, 2013.

[25] A. Recio-Spinoso and P. X. Joris, "Temporal properties of responses to sound in the ventral nucleus of the lateral lemniscus," Journal of Neurophysiology, vol. 111, no. 4, pp. 817-835, feb 2014.

[26] T. McKenna, J. Davis, and S. F. Z. and, Eds., Single Neuron Computation, ser. Neural Networks: Foundations to Applications. Elsevier, 1992.

[27] C. Koch and I. Segev, "The role of single neurons in information processing," Nature Neuroscience, vol. 3, no. S11, pp. 1171-1177, nov 2000.

[28] M. Nahmias, B. Shastri, A. Tait, and P. Prucnal, "A leaky integrate-andfire laser neuron for ultrafast cognitive computing," IEEE J. Sel. Topics Quantum Electron., vol. 19, no. 5, pp. 1-12, 2013.

[29] F. Selmi, R. Braive, G. Beaudoin, I. Sagnes, R. Kuszelewicz, and S. Barbay, "Temporal summation in a neuromimetic micropillar laser," Opt. Lett., vol. 40, no. 23, pp. 5690-5693, Dec 2015. [Online]. Available: http://ol.osa.org/abstract.cfm?URI=ol-40-23-5690

[30] B. Garbin, J. Javaloyes, G. Tissoni, and S. Barland, "Topological solitons as addressable phase bits in a driven laser," Nat Commun, vol. 6, pp. -, jan 2015. [Online]. Available: http://dx.doi.org/10.1038/ncomms6915

[31] B. Romeira, R. Avó, J. M. L. Figueiredo, S. Barland, and J. Javaloyes, "Regenerative memory in time-delayed neuromorphic photonic resonators," Scientific Reports, vol. 6, no. 1, jan 2016.

[32] S. Terrien, B. Krauskopf, N. G. R. Broderick, R. Braive, G. Beaudoin, I. Sagnes, and S. Barbay, "Pulse train interaction and control in a microcavity laser with delayed optical feedback," Opt. Lett., vol. 43, no. 13, p. 3013, jun 2018.

[33] S. Terrien, B. Krauskopf, N. G. R. Broderick, L. Andréoli, F. Selmi, R. Braive, G. Beaudoin, I. Sagnes, and S. Barbay, "Asymmetric noise sensitivity of pulse trains in an excitable microlaser with delayed optical feedback," Phys. Rev. A, vol. 96, no. 4, oct 2017.

[34] A. A. Faisal, L. P. J. Selen, and D. M. Wolpert, "Noise in the nervous system," Nature Reviews Neuroscience, vol. 9, no. 4, pp. 292-303, apr 2008.

[35] W. Maass, T. Natschläger, and H. Markram, "Real-time computing without stable states: A new framework for neural computation based on perturbations," Neural Computation, vol. 14, no. 11, pp. 2531-2560, nov 2002.

[36] B. Kelleher, C. Bonatto, P. Skoda, S. P. Hegarty, and G. Huyet, "Excitation regeneration in delay-coupled oscillators," Physical Review E, vol. 81, no. 3, mar 2010.

[37] T. V. Vaerenbergh, K. Alexander, J. Dambre, and P. Bienstman, "Excitation transfer between optically injected microdisk lasers," Optics Express, vol. 21, no. 23, p. 28922, nov 2013.
[38] M. Bache, F. Prati, G. Tissoni, R. Kheradmand, L. Lugiato, I. Protsenko, and M. Brambilla, "Cavity soliton laser based on vcsel with saturable absorber," Appl. Phys. B, vol. 81, pp. 913-920, 2005.

[39] S. Barbay, I. Sagnes, R. Kuszelewicz, and A. M. Yacomotti, "Localized states and excitability in a monolithic VCSEL with saturable absorber," in 2011 Fifth Rio De La Plata Workshop on Laser Dynamics and Nonlinear Photonics. IEEE, dec 2011.

[40] W. W. Chow, S. W. Koch, and M. Sargent, Semiconductor-Laser Physics. Springer Berlin Heidelberg, 1994.

[41] F. Selmi, "Excitable response and neuronlike properties in micropillar lasers with saturable absorber," Theses, Université Paris Sud - Paris XI, Sep. 2015. [Online]. Available: https://tel.archives-ouvertes.fr/ tel-01199595

[42] A. Rodan and P. Tino, "Minimum complexity echo state network," IEEE Transactions on Neural Networks, vol. 22, no. 1, pp. 131-144, jan 2011.

[43] G. M. Shepherd and R. K. Brayton, "Logic operations are properties of computer-simulated interactions between excitable dendritic spines," Neuroscience, vol. 21, no. 1, pp. 151-165, apr 1987.

[44] Á. Tóth and K. Showalter, "Logic gates in excitable media," The Journal of Chemical Physics, vol. 103, no. 6, pp. 2058-2066, aug 1995.

[45] R. Toth, C. Stone, B. de Lacy Costello, A. Adamatzky, and L. Bull, "Simple collision-based chemical logic gates with adaptive computing," in Theoretical and Technological Advancements in Nanotechnology and Molecular Computation. IGI Global, pp. 162-175.

[46] A. Jacobo, D. Gomilla, P. Colet, and M. Matias, "Logical operations using excitable cavity solitons," in CLEO/Europe - EQEC 2009 . European Conference on Lasers and Electro-Optics and the European Quantum Electronics Conference. IEEE, jun 2009.

[47] A. Jacobo, D. Gomila, M. A. Matìas, and P. Colet, "Logical operations with localized structures," New Journal of Physics, vol. 14, no. 1, p. 013040, 2012. [Online]. Available: http://stacks.iop.org/1367-2630/14/ $\mathrm{i}=1 / \mathrm{a}=013040$

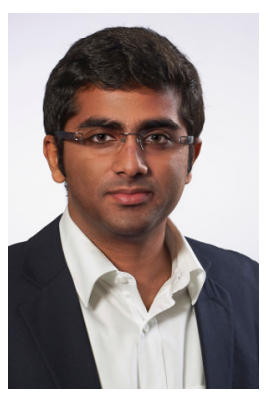

V. Anirudh Pammi V. Anirudh Pammi is currently a 2nd year PhD student at Centre de Nanosciences et de Nanotechnologies, Palaiseau. He received his undergraduate training in Electronics engineering from India and a joint masters in optics and photonics from Aix Marseille University and Karlsruhe Institute of Technology. His main research interests include integrated optics and novel substrates for computation.

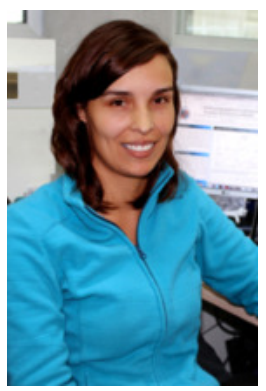

K. Alfaro-Bittner K. Alfaro-Bittner is currently a Ph.D. student at the Pontificia Universidad de Católica de Valparaíso. She received her undergraduate degree in Physics from Pontificia Universidad de Católica de Valparaíso in 2013. Her main research interests are nonlinear dynamical systems, particularly robust phenomena in nonlinear optics, liquid crystals, fluids, photonics, among others. 


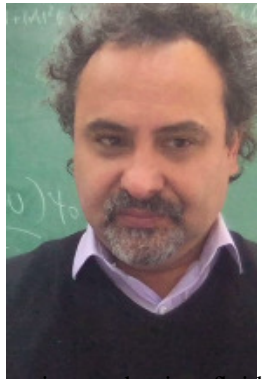

M. G. Clerc M. G. Clerc is a full professor of Physics at the Universidad de Chile, Chile. He received his undergraduate and master's degrees in Physics at the Universidad de Chile, in 1994 and 1996, and his Ph.D. in Physics from the University of Nice and Sophia-Antipolis, France, in 1999, followed by his postdoctoral appointment at the California Institute of Technology, CALTECH, USA. His research interests are focused on understanding the robust phenomena of non-equilibrium systems, which include soft matter, liquid crystals, nonlinear optic, mechanics, fluid dynamics, magnetics, granular media, population dynamics, statistical mechanics, stochastic processes, bifurcation, and dynamical systems theory.

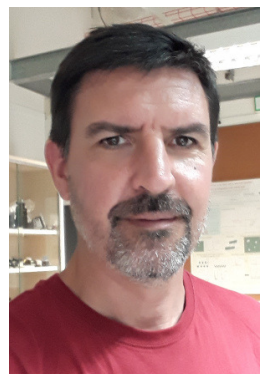

S. Barbay Sylvain Barbay is currently CNRS researcher and deputy manager of Photonics Department at the Centre for Nanoscience and Nanotechnology $(\mathrm{C} 2 \mathrm{~N})$ in Palaiseau, France. He received his $\mathrm{PhD}$ in physics from the University of Nice in 1998, and was then awarded a Marie-Curie postdoctoral fellowship at the Istituto Nazionale di Ottica (Florence, Italy). He then joined CNRS first at the Laboratoire de Photonique et de Nanostructures in Marcoussis (France) and then at the $\mathrm{C} 2 \mathrm{~N}$ in Palaiseau since 2016. His main research interests concern nonlinear dynamics in semiconductor micro and nano-cavities, and include neuromimetic photonics, spatiotemporal dynamical phenomena, localized structures and noise induced phenomena. 